than the essentials for either senior medical students or postgraduates, despite what the authors say in the preface. This is now a text book of clinical endocrinology, and none the worse for that.

In its approach to each topic, the text reflects the broad intellectual base of the authors. Thus good accounts of the biochemical and physiological actions of each gland are given before the clinical disorders and their treatment are discussed. The unknowns and currently incomprehensibles are not brushed unthoughtfully under the carpet. Good use of tables and line drawings is made. Although photographs of patients with gross endocrinological diseases "which have decorated endocrinology texts from time immemorial' have rightly been eschewed, some photographs would have been helpful, for example in describing the ophthalmic features of Graves' disease.

The balance of the book is in general good, although one might wish that the section on delayed puberty, which is common, was more proportional to precocious puberty, which is uncommon even if interesting. Indeed, this short section on delayed puberty brings me to my only criticism of this book - a criticism directed more at the publisher than the authors. Doubless for good (economic?) reasons the format in this third edition has undergone a radical change, to my eye for the worse. The former double column page was much easier to read and aesthetically more attractive. Delayed puberty in $\mathbf{4 5}$ lines of unbroken print is not a happy sight. It is hard to believe there will be a better book on endocrinology, except the next undoubted edition. Could we next time please have it back in its old format?

\section{Obstetrics by Ten Teachers}

Edited by S. G. Clayton, T. L. T. Lewis and G. Pinker. 13th edn. Pp. $x+541$, illustrated. Edward Arnold, London, 1980. $£ 13.50$.

'Ten teachers' occupies a special place in the range of obstetric text books, not least because of its long and distinguished pedigree. This, the thirteenth edition, has been due for some time - the last edition was produced in 1972. It is recognizable as soon as one opens the book, but it has undergone a considerable 'face-lift'. The paper is more pleasing than the old clay paper and the typeface and layout have been altered to good effect. A random index check was faultless, apart from a reference omission on p. 411, and the illustrations, particularly the X-ray and ultrasound photographs, are excellent.

The approach of this book is essentially 'classical' - this is both a strength and a weakness. It makes the chapters on standard aspects of obstetric practice strong, but weakness is shown on the more modern aspects of obstetric care. The chapters on placental insufficiency and prematurity are disappointing. These are major aspects of modern care, and deserve better cover. Not enough flexibility has been displayed in blending the various aspects of care and describing the attitude of mind of the clinician managing these conditions. However, at least they have been given the prominence of individual chapters.

'Ten Teachers' now occupies an awkward situation - between the 'pocket', note-like, inexpensive books and the many specialist texts. Its strength in the physiology and well established aspects of obstetrics make it a good introduction to the subject for the undergraduate and Diploma candidate. The editors see this as the book's role, and it fills it admirably.

One could find fault with some technical features in the content of the text. Growth retardation is not quoted as a cause of oligohydramnios; a true conjugate of down to $10 \mathrm{~cm}$ is cited as suitable for vaginal breech delivery - the importance of keeping the fetal back uppermost is not stressed in describing breech delivery. Pre-term labour is still called 'premature' labour; placental disease is not stresse疋 the list of causes. The subject is dealt with in 4 pages - fess space than that allotted to destructive operations on the feftus. I think most authorities would take up the statement that a bradycardia, rather than tachycardia, is one of the nost serious features of an abnormal computerized tocogram and the erroneous expression 'beat-to-beat' is perpetuated.

In dealing with epidural analgesia an increased forceps Fite is stated as being inevitable without explaining how it maybe minimized and lignocaine is stated as being the normal aggnt used. In describing caudal analgesia no mention is madef the field-rendered analgesic. External cephalic versior 5 is given considerable stress without putting it into its lesser role in the modern management of breech presentation. Inadvertent pre-term delivery is not quoted as a risk- of induction and maternal hypoxia is not cited as a riskorf general anaesthesia in obstetrics. Warfarin is stressed ascan anticoagulant with no mention of subcutaneous heparifoas an alternative. There is some evidence of weakness in the cross-referencing between chapters - thus a reader of the section on forceps does not encounter 'trial of forceps' andits essential role-but 'trial' is mentioned in discussing difsproportion. The chapter on fetal distress in labour is. Pstricted to describing cumulative, placental hypoxia. In this there is a danger; the 2 classical causes of distress, placental separation and cord presentation, are not mentioned in tFis chapter.

These points should not detract from a successful attempt to produce a new edition. There is so much of modern obstetrics open to healthy debate that any text book of this size cannot cover all technical or philosophical approaches.

\section{Pathology of the Liver}

Edited by R. N. M. MacSweEn, P. P. ANthonY P. J. Scheuer. Foreword by Hans Popper. Pp. 458, ill trated. Churchill Livingstone, Edinburgh, London aRुd New York, 1979. £27.00.

This is a multi-author text covering the whole spectrum of liver disease. All contributors are recognized internation authorities. As pointed out in the preface the book is $\frac{9}{n}$ extension to Professor Scheuer's well known introductofy monograph. There is a wealth of up-to-date information to be found in every chapter, invaluable to all specialists in the field. The editorial policy appears to have been rigorous that there is little overlap between different chapters. The reviewer found those on cirrhosis, primary biliary cirrhosis and liver pathology associated with diseases of other orgañs particularly lucid and enlightening. A little disappointing was that a mere 10 pages were given to the important subject Qf chronic hepatitis with no specific mention being made of the lesion commonly found in drug addicts. One of the introductory chapters deals with the pathophysiology of liver disease. Although of interest it is difficult to see how the fits in to the book which is largely concerned with histe. pathology.

\section{Surgical Treatment}

By Sir Charles Illingworth. Pp. 336, illustrate Pitman Medical, Tunbridge Wells, Kent, 1980. $£ 12.95$.

Sir Charles Illingworth, Emeritus Professor of Surgery in the University of Glasgow, can undoubtedly claim to be the doyen of the academic surgeons of the United Kingdom His pupils hold Chairs of Surgery throughout the Englisis speaking world and his textbooks still remain as standarg tomes on surgery and surgical anatomy. In his active retire ment he has how produced a concise volume on surgic 
practice which emphasizes modern methods of laboratory investigation and diagnosis as well as the principles of treatment and basic operative technique. The whole of surgery is covered in just over 300 pages, and this includes sections on fractures, cold orthopaedics, chest surgery and urology. Naturally this can only be achieved by keeping the text in a pithy and abbreviated form (for which Sir Charles is rightly famous) but this must also involve sacrifice. Thus, there is no discussion on anti-oestrogen drugs in the treatment of advanced breast cancer nor is there mention of the place of thyroid antibodies and the question of autoimmune disease in the section of Hashimoto's disease. However, there are numerous references for further reading so that additional information can be obtained where necessary.

Certainly this will prove to be a useful revision book for clinical students and its clear English will also recommend it to overseas doctors who plan to do postgraduate work in this country.

\section{Letter to the Editor}

March 23rd, 1981

\section{Treatment of pain in the elderly}

Dear Sir,

Our attention has been drawn to the letter from Dr S. Aron of Winthrop Laboratories, in the October 1980 issue of your Journal, commenting upon our article on the use of meptazinol, pentazocine and placebo in the treatment of pain in the elderly (Pearce and Robson, 1980).

Dr Aron's criticism of the dose of pentazocine chosen for this trial evidently results from insufficient attention to the introduction to the paper.

It is clearly stated therein that the equivalence in analgesic potency of meptazinol $100 \mathrm{mg}$ to pentazocine $60 \mathrm{mg}$ refers to administration by the intramuscular route. Meptazinol, in common with many other analgesics, is subject to an important hepatic first pass phenomenon (Rosseel et al., 1976) following oral administration, and higher relative doses are, therefore, required by this route. Thus meptazinol $100 \mathrm{mg}$ is the minimum recommended dose by the oral route and, according to MIMS, the same applies to pentazocine $25 \mathrm{mg}$. The selection of these doses is borne out by the fact that meptazinol $200 \mathrm{mg}$ orally has been shown to be equianalgesic to pentazocine $50 \mathrm{mg}$ orally in patients suffering from chronic rheumatoid or osteoarthritis (FlavellMatts and Ward, 1980).

The reasons for deliberately choosing small doses in the elderly population are also clearly stated in the introduction: age is highly correlated with the pain relief obtained from a given dose of analgesic (Bellville et al., 1971) and the elderly are more susceptible to drugs in general (Editorial, 1977). It, therefore, seemed logical to compare the smallest recommended doses of the two analgesics with placebo in this population whose mean age was $81.4 \pm 6.4$ years.

Dr Aron's comments on side effects seem somewhat specious when such a small incidence was noted in all three treatment groups.

We would not disagree with Dr Aron's claim that pentazocine may be a useful medicine in the elderly, and in this trial it was clearly distinguishable from placebo. However, meptazinol has significant advantages over pentazocine for this group of patients.

Royal Devon and Exeter Hospital (Heavitree),

Yours faithfully, V. PearCe, M.B., M.R.C.P., $\quad$ P. J. Robson, M.B., M.R.C.P.

Gladstone Road,

Exeter EX1 2ED.

\section{References}

Bellville, J.W., Forrest, W.H., Miller, E. \& Brown, B.W. (1971) Influence of age on pain relief from analgesics: A study of post-operative patients. Journal of the American Medical Association, 217, 1835.

EDITORIAL (1977) Drugs and the elderly. Lancet, ii, 693.

Flavell-Matts, S.G. \& WARD, P.J. (1980) A double blind comparison of meptazinol versus pentazocine in chronic rheumatoid and osteoarthritis. British Journal of Clinical Practice, 34, 286.

Pearce, V. \& Robson, P.J. (1980) Double-blind crossover trial of oral meptazinol, pentazocine and placebo in the treatment of pain in the elderly. Postgraduate Medical Journal, 56, 474.

Rosseel, M.T., Bogaert, M.G., Verschraegen, R. \& StaQuet, M. (1976) Plasma concentrations of meptazinol (Wy 22811) after different routes of administration in man. Pharmatherapeutica, 1, 270. 